\title{
Variable sensitivity and specificity of TTF-1 antibodies in lung metastatic adenocarcinoma of colorectal origin
}

\author{
Eva Compérat ${ }^{1}$, Fan Zhang ${ }^{1}$, Cedric Perrotin ${ }^{2}$, Thierry Molina ${ }^{1}$, Pierre Magdeleinat ${ }^{2}$, \\ Beatrice Marmey ${ }^{1}$, Jean-Francois Régnard ${ }^{2}$, Josee Audouin ${ }^{1}$ and Saphie Camilleri-Broët ${ }^{1}$ \\ ${ }^{1}$ Service d'Anatomopathologique de l'Hôtel-Dieu, place du Parvis Notre Dame, Paris, France and ${ }^{2}$ Service de \\ chirurgie thoracique de l'Hôtel-Dieu, place du Parvis Notre Dame, Paris, France
}

\begin{abstract}
Thyroid transcription factor-1 (TTF-1) is considered as a reliable marker for differential diagnosis in distinguishing primary adenocarcinomas of the lung from extrathoracic origins. We previously reported the first case of lung metastasis of colorectal origin, with nuclear expression of TTF-1. As most previous studies were performed on series of extrathoracic primary tumors, we raised the question of a possible role of lung microenviroment in TTF-1 expression. We investigated the rate of TTF-1 expression in lung metastases of extrathoracic adenocarcinomas and compared results of immunohistochemistry performed with different primary antibodies. Two different clones of antibodies (8G7G1/1 from Dako, SPT24 from Novocastra) raised against TTF-1 were used on 56 lung-metastatic malignant tumors, 41 from colorectal origin. A series of primary colorectal (90 cases) and primary pulmonary adenocarcinomas (86 cases) were also investigated. Four of 41 $(10 \%)$ lung metastases of colorectal adenocarcinomas displayed a nuclear staining for TTF-1 with SPT24 clone. Three of the four positive cases displayed similar nuclear staining in primary and/or other extrathoracic metastatic sites as well as four of $90(5 \%)$ primary colorectal adenocarcinomas, ruling out the role of lung microenvironment. None of them was positive with 8G7G1/1 clone. Sensitivity between two sets of antibodies was compared in 86 primary pulmonary adenocarcinomas. Nuclear staining was detected in 72 cases (84\%) with Novocastra's antibody and 56 cases $(65 \%)$ with Dako's. Significant discordance was observed $(P<0.01)$. These results suggest that the diagnostic virtue of TTF- 1 detection depends on the used antibody's clone. The SPT24 clone seems to have a stronger affinity for TTF-1 protein but may lead to a few positive colorectal adenocarcinomas.
\end{abstract}

Modern Pathology (2005) 18, 1371-1376. doi:10.1038/modpathol.3800422; published online 29 April 2005

Keywords: TTF-1; lung; carcinoma; metastasis; colorectal

Thyroid transcription factor-1 (TTF-1) is a tissuespecific transcription factor expressed in the epithelial cells of thyroid and lung (type II pneumocytes and Clara cells). Carcinomas arising from lung and thyroid also show frequent TTF-1 expression. ${ }^{1,2}$ As the lung is one of the most common sites of metastasis, TTF-1 is considered as a reliable marker to distinguish between primary lung carcinoma and lung metastasis, especially when dealing with an adenocarcinoma or a large-cell carcinoma. ${ }^{3-6}$ It is also considered as a reliable marker in the differential diagnosis between pleural localization of lung

Correspondence: Dr S Camilleri-Broët, MD, Service d'Anatomopathologique de l'Hôtel-Dieu, 1, place du Parvis Notre Dame, 75181 Paris Cedex 04, France.

E-mail: anapath.hd@htd.aphp.fr

Received 23 November 2004; revised and accepted 14 December 2004; published online 29 April 2005 origin carcinoma and malignant mesothelioma. Two main commercial available clones of monoclonal antibodies have been raised against TTF-1 for immunohistochemical use, 8G7G1/1 and SPT24, their diagnostic value in determining the lung or thyroid origin of adenocarcinoma has not yet been compared. Most of the studies show the high specificity of TTF-1 detection and have been published used the $8 \mathrm{G} 7 \mathrm{G} 1 / 1$ clone. We previously reported one case of lung metastasis of colorectal origin, which showed a focal nuclear staining with the anti-TTF-1 antibody (clone SPT24) on both paraffin-embedded and cryostat sections. ${ }^{8}$ To our knowledge, this was the first TTF-1-positive colorectal carcinoma reported.

In this study, we investigated a series of metastatic malignant tumors in the lung to detect the rate of TTF-1-positive lung metastases. Our results showed surprisingly a non-negligible rate of TTF-1-positive 
metastatic adenocarcinomas of colorectal origin. This result differed from most published studies showing TTF-1 as a specific marker of primary lung or thyroid origin when dealing with adenocarcinoma or large-cell carcinoma. As most of the previous studies were performed on primary colonic adenocarcinomas instead of their lung metastatic location, we raised the question of a role of the lung microenviroment in the induction of such an expression. To answer this question, we investigated the primary colonic tumors and/or other metastatic sites in all the TTF-1-positive cases as well as a large series of primary colonic adenocarcinomas. Additionally, we investigated and compared the results obtained with the two main antibodies used in routine (clone 8G7G1/1 and SPT24) raised against TTF-1 protein.

\section{Materials and methods}

\section{Materials}

A total of 232 tumors, including 56 lung metastatic tumors, 90 primary colonic adenocarcinomas, with typical, localization (all were found colic surgical samples) and histology, and 86 primary pulmonary adenocarcinomas, were retrospectively collected from the files of the department of Pathology, Hôtel-Dieu Hospital, Paris. Patients underwent surgical resection between September 1997 and September 2003, including 144 male and 88 female patients. The mean age of patients was 65 years (range 17-97 years). For the metastatic tumors located in the lung, most cases corresponded to colorectal origin (41 cases), the others originated from kidney (six cases), breast (two cases), liver (two cases), sarcoma (three cases), salivary glands (one case) or thyroid (one case). For the TTF-1-positive metastatic tumors, primary tumors as well as lymph nodes or liver metastases were also analyzed when available.

\section{Tissue Arrays Construction}

Tissue microarrays blocks were assembled by using a manual 24-well arrayer (Histopathology Ltd., Pécs, Hungary). The fields of tumor for tissue microarrays construction were selected through microscopic examination on the corresponding sections. For each case, two cores of $2 \mathrm{~mm}$ diameter were punched. Sections of $3 \mu \mathrm{m}$ thickness were carefully cut from tissue microarray blocks, and the first slide was hematoxylin-eosin (H\&E)-stained to verify adequate representation of diagnostic areas.

\section{Immunohistochemistry}

Immunohistochemistry for TTF-1 expression was performed either on conventional slides at the time of diagnosis (for metastatic lung tumors) or retro- spectively on tissue microarrays sections. The slides were deparaffinized in xylene and rehydrated through graded alcohols to water. Antigen retrieval was performed by microwave of tissue sections in $10 \mathrm{mM}$ sodium citrate buffer $(\mathrm{pH} 6.0)$ for $15 \mathrm{~min}$ at $750 \mathrm{~W}$. Two different clones raised against TTF-1 were used: clone SPT24 (Novocastra, UK, 1/100 dilution) and clone 8G7G3/1 (Dako, USA, 1/50 dilution). After $60 \mathrm{~min}$ incubation with the primary antibody, a standard streptavidin-biotin-peroxidase complex (SABC) method was applied (ABCYS GMR4-61; BIOSPA, Milano, Italy), with $15 \mathrm{~min}$ incubation for both second antibody and SABC complex. The staining was visualized with $3,3^{\prime}$ diaminobenzidine tetrahydrochloride (DAB) and the slides were counterstained with Mayer's hematoxylin. Lung parenchyma adjacent to tumor was used as internal positive control. The slides were examined by two pathologists and were scored as following: 0 when no expression or faint expression of $<5 \%$ of the tumor nuclei, 1 when faint expression of $\geq 5 \%$ of the tumor nuclei, 2 when bright expression of $\geq 5 \%$ of the tumor nuclei. 1 and 2 were considered as positive staining. Cytoplasmic staining was considered nonspecific. For TTF-1-positive cases of lung metastases from colonic adenocarcinoma, the CK7 and CK20 expressions in tumoral cells (anti-CK7, anti-CK20: 1/50 dilution; DAKO, USA) were also tested.

\section{Statistical Analysis}

The rates of TTF-1 positivity between metastatic and primary colorectal adenocarcinomas were analyzed by Yates' $\chi^{2}$ method, and the agreement between two sets of primary antibody on the lung-originated adenocarcinomas was analyzed by McNemar method.

\section{Results}

\section{TTF-1 Expression of Lung Metastatic Tumors}

Out of 56 cases studied on standard sections with the primary antibody from Novocastra (clone SPT24), five cases were considered positive for TTF-1. One case was of thyroid origin and four cases were of colorectal origin. For the four lung metastases of colorectal adenocarcinomas, the diagnosis of colorectal origin was unequivocal, based on clinical history, the presence of several lung nodules, the typical histological features of colorectal origin with polyadenoid patterns (all were well-differentiated adenocarcinomas) and the immunoprofile was CK7-/CK20 + (Table 1). For these four positive cases, the nuclear staining of TTF-1 was focal, corresponding to $10-40 \%$ of the surface analyzed. Some tumour glands displayed positivity in all the cells whereas others showed partial positivity (Figure 1). Cytoplasmic immunostaining 
Table 1 Immunohistochemical profile of primary colorectal cancer and metastasis

\begin{tabular}{|c|c|c|c|c|}
\hline Case & $\begin{array}{l}\text { Primary colorectal tumor/ } \\
\text { CK } 20 / C K 7\end{array}$ & $\begin{array}{l}\text { Primary colorectal tumor, } \\
\text { TTF-1(clone SPT24) }\end{array}$ & $\begin{array}{l}\text { Lung metastatic } \\
\text { immunoprofile }\end{array}$ & $\begin{array}{l}\text { Other metastatic site, } \\
\text { TTF-1(clone SPT24) }\end{array}$ \\
\hline 1 & CK20+/CK7- & TTF1+ & TTF1+,CK20+/CK7- & Liver, TTF-1+ \\
\hline 2 & CK20+/CK7- & TTF $1+$ & TTF-1+,CK20+/CK7- & Liver, TTF-1+ \\
\hline 3 & Not available & Not available & TTF-1+,CK20+/CK7- & Lymph node, TTF-1+ \\
\hline 4 & CK20+/CK7- & $\begin{array}{l}\text { TTF-1-(fixed in Bouin's } \\
\text { liquid) }\end{array}$ & TTF-1+,CK20+/CK7- & None \\
\hline
\end{tabular}

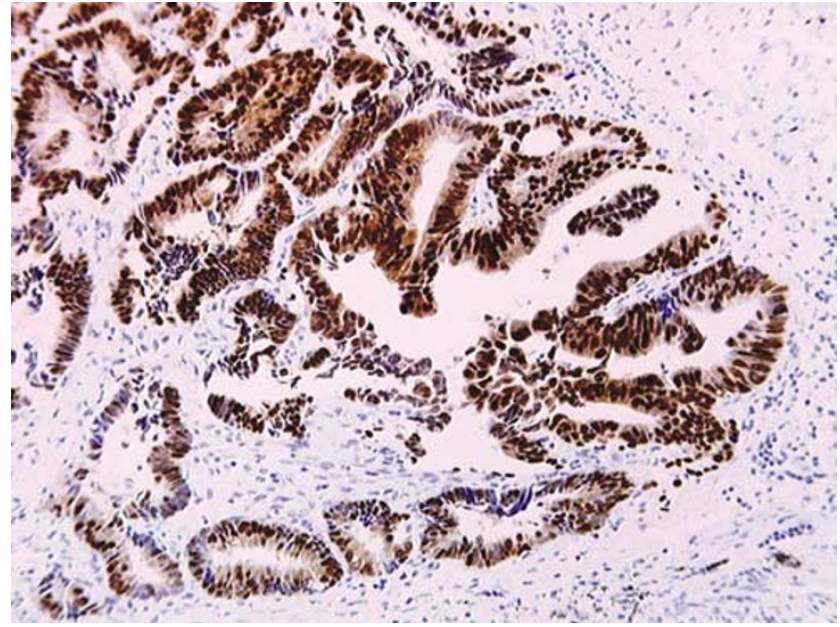

Figure 1 Lung metastasis: Novocastra antibody clone SPT24.

was associated with nuclear staining in two cases. In some areas, the intensity of nuclear staining in tumor cells was similar to that in adjacent normal pneumocytes.

In order to investigate the potential microenvironmental role for such an expression, we analyzed retrospectively at least one other tumor site for the four positive cases. The primary colorectal carcinomas were available in three cases, a metastatic mesenteric lymph node in one case and liver metastasis in two cases. In three of the four positive cases, focal moderate to bright nuclear staining was seen in the tested extrathoracic sites for TTF-1 analyzed with the same antibody (clone SPT24) (Figure 2). The last case was negative for TTF-1 in the primary site, but the sample was fixed in Bouin's liquid and contained a few number of tumor glands within a large area of necrosis.

When using the primary antibody from Dako (clone 8G7G3/1), the four positive lung metastases, as well as their primary adenocarcinomas, were negative (Figure 3). However, in one case, a very slight nuclear staining (gray) was observed in a few cells located in the same positive area detected with Novocastra's antibody, but the intensity and the number of stained cells was found insufficient to be scored as positive. Both antibodies successfully revealed the TTF-1 expression in normal pneumocytes adjacent to the metastatic tumors, but the

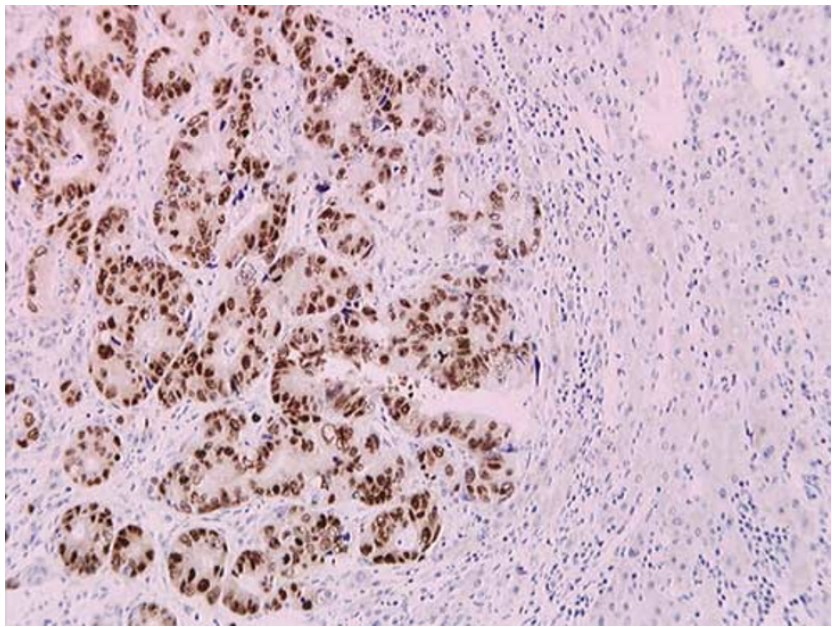

Figure 2 Liver metastasis: Novocastra antibody clone SPT24.

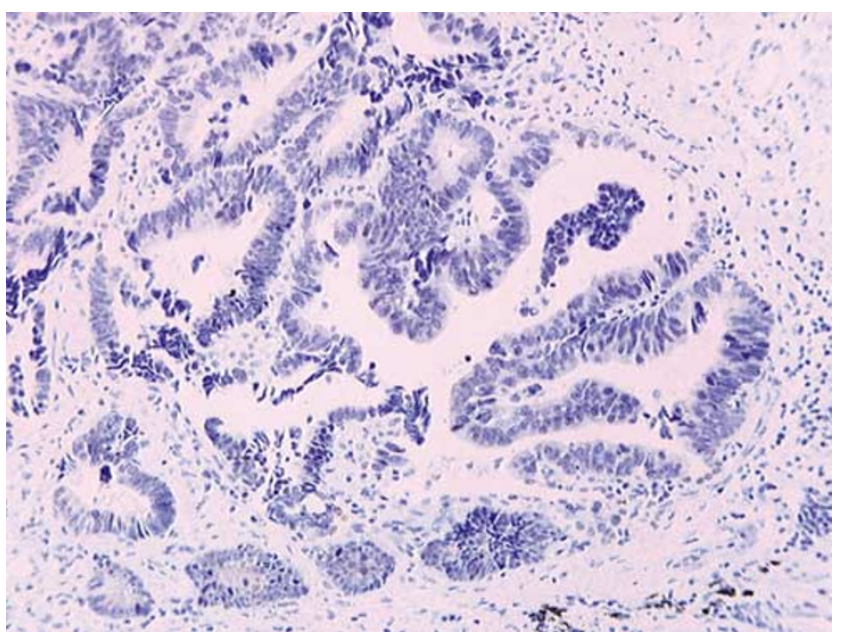

Figure 3 Lung metastasis: Dako antibody 8G7G3/1.

staining with Novocastra's antibody was much stronger than that with Dako's.

\section{TTF-1 Expression of Primary Colorectal Adenocarcinomas}

Four of the 90 (5\%) examined cases on tissue microarray sections showed nuclear staining for 


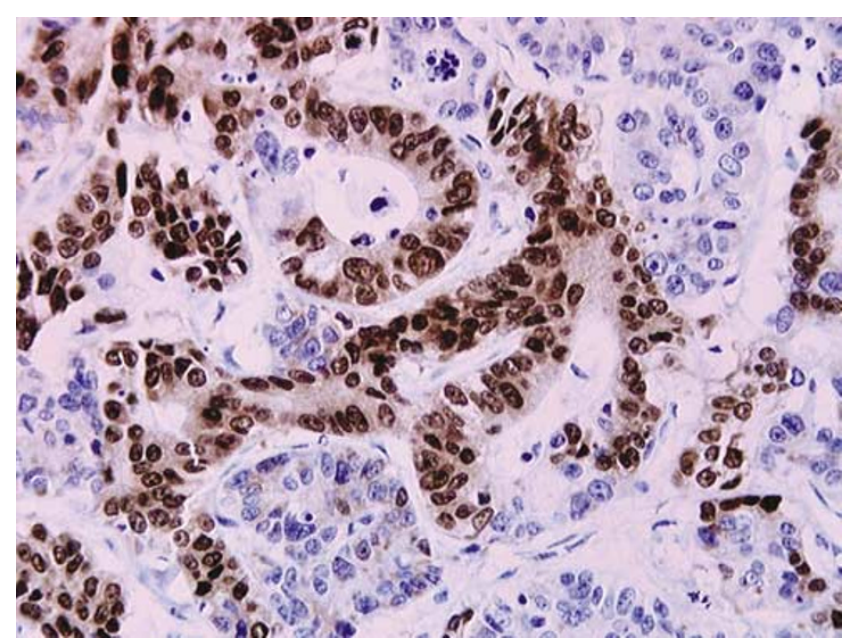

Figure 4 Colon carcinoma: Novocastra antibody clone SPT24.

TTF-1 using Novocastra's antibody (clone SPT24) (Figure 4). These cases were identified by grossly and histologically findings as primary colorectal carcinomas with CK20 + / CK7 - immunoprofile. All these patients underwent pulmonary scanner before surgery, none displayed any lung metastasis at that time. Similar to positive lung metastases, nuclear staining was focal accounting $20-40 \%$ of the surface analyzed, with moderate to high intensity and often associated with cytoplasmic staining. The positive rate in colorectal adenocarcinomas was not significantly different to that in lung metastatic tumors.

In contrast, none of the 90 cases of primary colorectal adenocarcinoma was positive when using Dako's antibody.

\section{TTF-1 Expression of Primary Pulmonary Adenocarcinomas}

In the 86 tested cases, 72 cases (84\%) were TTF-1 positive with Novocastra's antibody and 56 cases $(65 \%)$ showed positivity with Dako's antibody. The two antibodies were concordant in 56 positive cases and 14 negative cases. However, 16 cases (19\%) were detected positive by Novocastra's antibody (Figure 5), but negative with Dako's antibody (Figure 6). Moreover, the intensities of nuclear staining were generally stronger with Novocastra's antibody than with Dako's, for the fact that 13 positive samples were scored as ' 2 ' by using the former and were scored as ' 1 ' by using the latter. A significant discordance between two sets of antibody was obtained in McNemar analysis $(P<0.01)$. The differences suggest a higher affinity of Novocastra's antibody for TTF-1 protein compared with Dako's.

\section{Discussion}

As lung parenchyma is a common site of metastases, the differential diagnosis between metastatic and

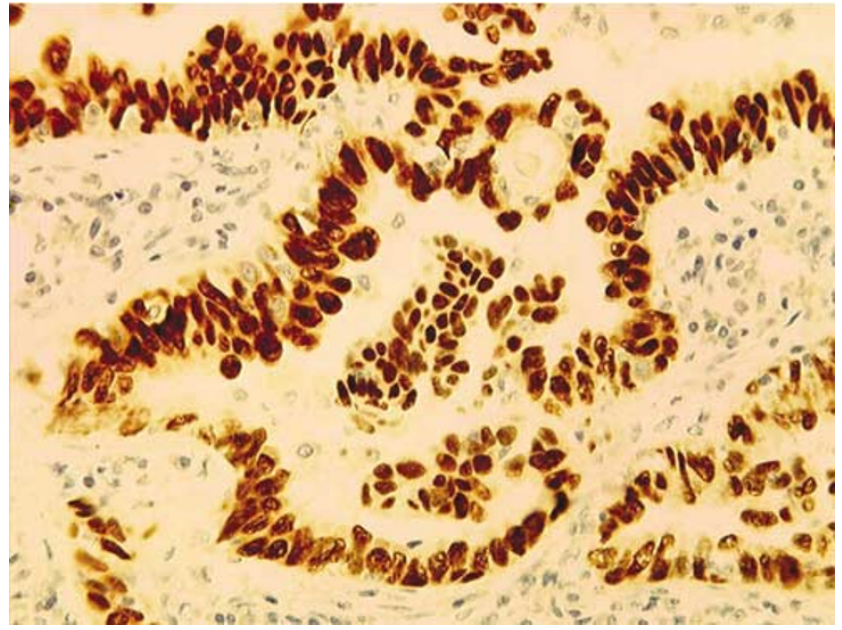

Figure 5 Primary lung adenocarcinoma: Novocastra antibody clone SPT24.

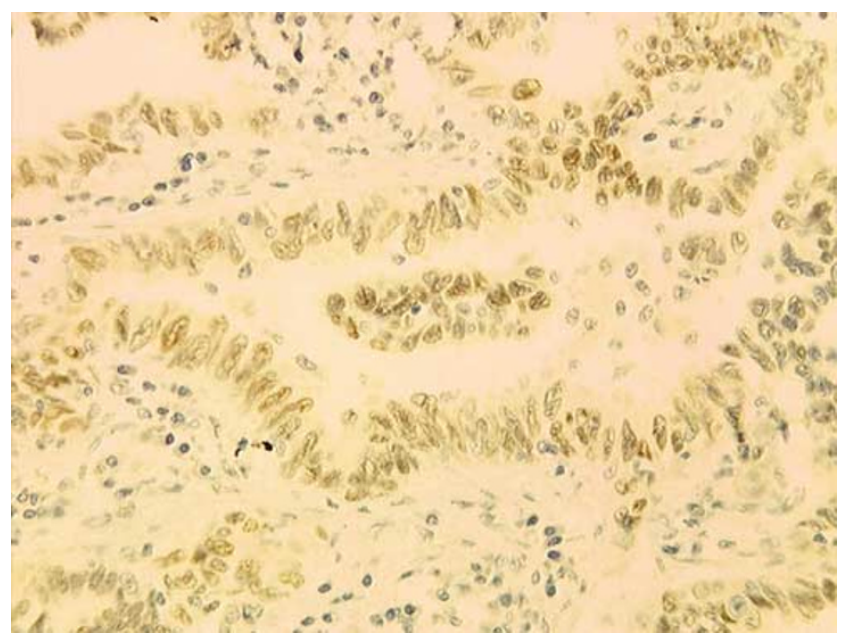

Figure 6 Primary lung adenocarcinoma: Dako antibody 8G7G3/1.

primary lung carcinoma is a frequent perplexity. Histological features may not be sufficient to permit unequivocal confirmation and additional reliable immunohistochemical markers are required in the differentiation of these malignancies. The immunohistochemical detection for the nuclear expression of TTF-1 is considered as a useful marker in favor of lung or thyroid origin because of its high specificity (95-100\%). ${ }^{9-12}$ In the literature, TTF-1 expression in nuclei has been shown in rare cases of adenocarcinomas arising outside from lung or thyroid: one weakly positive case of metastatic ovarian carcino$\mathrm{ma}^{13}$ and one focal positive case of endometrial carcinoma. ${ }^{14}$ Recently, our group has shown one positive adenocarcinoma of colorectal origins, ${ }^{8}$ whereas no such positivity was reported in literature (Table 2).

The aim of present study was to investigate the rate of TTF-1-positive lung metastases of extrathoracic tumors, the potential role of the lung microenviroment in such an expression and the 
Table 2 Reported TTF-1 expression in primary or metastatic colorectal carcinomas

\begin{tabular}{|c|c|c|c|c|c|c|c|}
\hline First author & Year & Type & Cases & $T T F-1(+)$ & Clone & Producer & Antigen retrieval \\
\hline Bejarano $^{14}$ & 1996 & Primary & 18 & 0 & Polyclonal & Authors & Microwave treating \\
\hline Holzinger ${ }^{15}$ & 1996 & Primary & 21 & 0 & $8 \mathrm{G} 7 \mathrm{G} 3 / 1$ & Authors & Microwave treating \\
\hline Ordonez $^{16}$ & 2000 & Primary & 10 & 0 & $8 \mathrm{G} 7 \mathrm{G} 3 / 1$ & Labvision & Streaming in citrate buffer \\
\hline Kaufmann ${ }^{3}$ & 2000 & Primary & 30 & 0 & 8G7G3/1 & Neomarkers & Pressure cooking in EDTA \\
\hline Chang $^{12}$ & 2004 & Primary & 15 & 0 & $8 \mathrm{G} 7 \mathrm{G} 3 / 1$ & Dako & Heating in citrate buffer \\
\hline Moldvay $^{4}$ & 2004 & Primary & 26 & 0 & $8 \mathrm{G} 7 \mathrm{G} 3 / 1$ & Dako & Microwave treating \\
\hline Reis-Filho ${ }^{10}$ & 2000 & Lung Metastatic & 3 & 0 & $8 \mathrm{G} 7 \mathrm{G} 3 / 1$ & Neomarkers & Microwave in citrate buffer \\
\hline Jang $^{5}$ & 2001 & Lung Metastatic & 8 & 0 & $8 \mathrm{G} 7 \mathrm{G} 3 / 1$ & Dako & Microwave in citrate buffer \\
\hline Chhieng $^{9}$ & 2001 & Lung Metastatic & 6 & 0 & 8G7G3/1 & Neomarkers & Microwave in citrate buffer \\
\hline $\mathrm{Ng}^{6}$ & 2002 & Lung Metastatic & 5 & 0 & 8G7G3/1 & Dako & Streaming in EDTA \\
\hline Wieczorek $^{17}$ & 2002 & Liver Metastatic & 26 & 0 & 8G7G3/1 & Dako & Heating in EDTA \\
\hline
\end{tabular}

sensitivity or specificity of TTF-1 detection when using the two main commercial antibodies. Our study showed that four cases of lung metastases of colorectal adenocarcinomas displayed a nuclear staining for TTF-1 when using Novocastra's antibody. The staining was usually focal and sometimes associated with cytoplasmic staining. The relatively high rate of expression in our study compared to the reported papers raised the question of its mechanism. A potential role of lung microenvironment was ruled out by demonstration of similar TTF-1 expression in primary colorectal tumors and/or other extrathoracic metastases for three of the four positive cases, showing a similar staining as that found in lung. Moreover TTF-1 staining was demonstrated in four of the 90 primary colorectal adenocarcinomas, slightly less frequent than that in lung metastatic carcinomas of colorectal origin (four of 41 cases). This difference was not statistically significant and probably due to the use of TMA technique on the former in contrast to the conventional sections used for lung metastases samples.

Most of the published studies used the $8 \mathrm{G} 7 \mathrm{G} 3 / 1$ clone for the detection of TTF-1 protein. The 8G7G1/1 monoclonal antibody ${ }^{15}$ had been raised by using a recombinant rat TTF-1 (more than 370 amino acids) as immunogen, since the human TTF-1 shared $98 \%$ homology with the equivalent rat and mouse proteins. The antibody of $8 \mathrm{G} 7 \mathrm{G} 1 / 1$ clone had been tested excellently specific for lung or thyroid origin adenocarcinoma and large-cell carcinoma, while it had also been reported positive in some non-lung origin small-cell carcinomas. ${ }^{16}$ This antibody has shown some nonspecific cytoplasmic staining and suspected to crossreact with a cytoplasmic protein in liver cells. ${ }^{17}$ In the current study, we also used another monoclonal antibody, the SPT24 clone, to detect the expression of TTF-1. In total, $10 \%$ of lung-metastatic colorectal adenocarcinoma and $5 \%$ of primary colorectal adenocarcinomas were found positive, respectively, these results dramatically differ from the literature, which showed no positive cases in a total of 131 primary colonic carcinomas and 52 metastatic carcinomas in the lung (Table 1). When we tested positive lung metastatic and primary colonic adenocarcinoma with the 8G7G3/1 clone (Dako's) in dilution of $1 /$ 40 and $1 / 20$, no case showed a significant nuclear positivity. This difference in specificity according to the used antibodies raised two main hypotheses: Firstly, there may be a higher affinity of SPT24 clone for TTF-1, which aberrantly expressed with a low level in colorectal adenocarcinoma that it could not be detected by 8 G7G1/1 clone. Secondly, the antibody of SPT24 clone may have a crossreaction with another nuclear protein in some colorectal tumoral cells.

The SPT24 clone has been raised against a 123amino-acid fragment of the N-terminal region of human TTF-1 protein (gi:4507715, http://www.ncbi. nlm.nih.gov). As it is much shorter, the antibody raised using this immunogen may have less chance to acquire crossreaction. It has been reported that the antibody of SPT24 clone is superior for its consistent nuclear positivity and less erratic cytoplasmic staining. ${ }^{18}$ As for its good sensitivity, we have demonstrated that it could be applied on frozen-section diagnosis by using rapid immunohistochemical technique. ${ }^{8}$ In the present work, we noticed that the nuclear expression of TTF-1 in normal pneumocytes tested with SPT24 clone was stronger than that with $8 \mathrm{G} 7 \mathrm{G} 3 / 1$ clone. Also, a slight staining with Dako's antibody in 1/10 dilution (data not shown) could be observed in the same positive zone of a metastatic colorectal carcinoma detected with Novocastra's. Moreover, when comparing the immunostaining of immunoreaction of primary lung adenocarcinomas with these two antibodies, we showed that SPT24 clone was much more sensitive than $8 \mathrm{G} 7 \mathrm{G} 3 / 1$ clone with a positive rate of $84 \%$ in contrast to $65 \%$ to the latter. These results are in favor for a higher affinity of the SPT24 clone. It is possible that TTF-1 may be aberrantly expressed in a few colorectal carcinomas caused by some genetic alteration such as an amplification of the chromosomal region including the TTF-1 gene. However, the level of this aberrant expression may be lower than usual as in pneumocytes and only discerned by SPT24 clone with higher sensitivity. This difference in the reactivity of antibody's clones was also seen 
in employing E-cadherin immunostaining for distinguishing mesothelioma and pulmonary adenocarcinoma. ${ }^{19}$ Thus, we propose the attention on using different clones of antibody raised against the same protein for diagnostic purpose.

In conclusion, our study showed that the specificity of TTF-1 expression for attesting primary lung origin of adenocarcinoma depends partially of the clones of the employed antibody. The SPT24 clone has a stronger affinity for TTF-1 protein but may lead to a few TTF-1 positive colorectal adenocarcinomas. The testing of commercial antibodies from different clones is essential to assess their diagnostic virtue for differentiation use.

\section{Acknowledgements}

We thank P-E Bouchet, G Mangeon for section and S Bain, for their technical assistance. We also thank $C$ Guettier, B Terris and F Bendjaballah for providing paraffin embedded blocs of primary tumors of TTF-1 positive metastases. Thanks to J-F Emile for CK7/ CK20 immunostaining. We also thank G Szekeres for having kindly provided us the tissue arrayer.

\section{References}

1 Katoh R, Kawaoi A, Miyagi E, et al. Thyroid transcription factor-1 in normal, hyperplastic, and neoplastic follicular thyroid cells examined by immunohistochemistry and nonradioactive in situ hybridization. Mod Pathol 2000;13:570-576.

2 Stenhouse G, Fyfe N, King G, et al. Thyroid transcription factor 1 in pulmonary adenocarcinoma. J Clin Pathol 2004;57:383-387.

3 Kaufmann O, Dietel M. Thyroid transcription factor-1 is the superior immunohistochemical marker for pulmonary adenocarcinomas and large cell carcinomas compared to surfactant proteins A and B. Histopathology 2000;36:8-16.

4 Moldvay J, Jackel M, Bogos K, et al. The role of TTF-1 in differentiating primary and metastatic lung adenocarcinomas. Pathol Oncol Res 2004;10:85-88.

5 Jang KY, Kang MJ, Lee DG, et al. Utility of thyroid transcription factor- 1 and cytokeratin 7 and $20 \mathrm{im}-$ munostaining in the identification of origin in malignant effusions. Anal Quant Cytol Histol. 2001;23: 400-404.

6 Ng WK, Chow JC, Ng PK. Thyroid transcription factor-1 is highly sensitive and specific in differentiating metastatic pulmonary from extrapulmonary adenocarcinoma in effusion fluid cytology specimens. Cancer 2002;96:43-48.
7 Di Loreto C, Puglisi F, Di Lauro V, et al. TTF-1 protein expression in pleural malignant mesotheliomas and adenocarcinomas of the lung. Cancer Lett 1998;124: 73-78.

8 Camilleri-Broet $\mathrm{S}$, Alifano $\mathrm{M}$, Morcos $\mathrm{M}$, et al. Peroperative frozen section analysis of TTF-1 antigen expression. J Clin Pathol 2004;57:98-100.

9 Chhieng DC, Cangiarella JF, Zakowski MF, et al. Use of thyroid transcription factor 1, PE-10, and cytokeratins 7 and 20 in discriminating between primary lung carcinomas and metastatic lesions in fine-needle aspiration biopsy specimens. Cancer 2001;93:330-336.

10 Reis-Filho JS, Carrilho C, Valenti C, et al. Is TTF1 a good immunohistochemical marker to distinguish primary from metastatic lung adenocarcinomas? Pathol Res Pract 2000;196:835-840.

11 Abutaily AS, Addis BJ, Roche WR. Immunohistochemistry in the distinction between malignant mesothelioma and pulmonary adenocarcinoma: a critical evaluation of new antibodies. J Clin Pathol 2002;55: 662-668

12 Chang YL, Lee YC, Liao WY, et al. The utility and limitation of thyroid transcription factor-1 protein in primary and metastatic pulmonary neoplasms. Lung Cancer 2004;44:149-157.

13 Hecht JL, Pinkus JL, Weinstein LJ, et al. The value of thyroid transcription factor-1 in cytologic preparations as a marker for metastatic adenocarcinoma of lung origin. Am J Clin Pathol 2001;116:483-488.

14 Bejarano PA, Baughman RP, Biddinger PW, et al. Surfactant proteins and thyroid transcription factor-1 in pulmonary and breast carcinomas. Mod Pathol 1996;9:445-452.

15 Holzinger A, Dingle S, Bejarano PA, et al. Monoclonal antibody to thyroid transcription factor-1: production, chraracterization and usefulness in tumor diagnosis. Hybridoma 1996;15:49-53.

16 Ordonez NG. Value of thyroid transcription factor-1 immunostaining in distinguishing small cell lung carcinomas from other small cell carcinomas. Am J Surg Pathol 2000;24:1217-1223.

17 Wieczorek TJ, Pinkus JL, Glickman JN, et al. Comparison of thyroid transcription factor-1 and hepatocyte antigen immunohistochemical analysis in the differential diagnosis of hepatocellular carcinoma, metastatic adenocarcinoma, renal cell carcinoma, and adrenal cortical carcinoma. Am J Clin Pathol 2002; 118:911-921.

18 Pan CC, Chen PC, Tsay SH, et al. Cytoplasmic immunoreactivity for thyroid transcription factor- 1 in hepatocellular carcinoma: a comparative immunohistochemical analysis of four commercial antibodies using a tissue array technique. Am J Clin Pathol 2004;121:343-349.

19 Ordonez NG. Value of E-cadherin and N-cadherin immunostaining in the diagnosis of mesothelioma. Hum Pathol 2003;34:749-755. 